\title{
F sonstem \\ Pilot study of expanded newborn screening for 573 genes related to severe diseases in China: results from 1173 newborns
}

\section{Xiaomei Luo}

Shanghai Jiaotong University School of Medicine Xinhua Hospital

\section{Yu Sun}

Shanghai Jiaotong University School of Medicine Xinhua Hospital

\section{Feng Xu}

Shanghai Jiaotong University School of Medicine Xinhua Hospital

\section{Jun Guo}

Shanghai Jiaotong University School of Medicine Xinhua Hospital

\section{Lin Li}

Nanjing Novogene BioTechnologu Co.,Ltd

\section{Zhiwei Lin}

Nanjing novogene Biotechnology Co., Ltd

Jun Ye

Shanghai Jiaotong University School of Medicine Xinhua Hospital

\section{Xuefan Gu}

Shanghai Jiaotong University School of Medicine Xinhua Hospital

\section{Yongguo Yu ( $\nabla$ yuyongguo@shsmu.edu.cn )}

Xin Hua hospital affiliated to Shanghai Jiaotong University school of Medicine https://orcid.org/00000002-4472-0910

\section{Research article}

Keywords: expanded newborn screening, next-generation sequencing, birth defect, carrier frequencies, inherited metabolic disease

Posted Date: August 21st, 2019

DOI: https://doi.org/10.21203/rs.2.13332/v1

License: (c) (1) This work is licensed under a Creative Commons Attribution 4.0 International License. Read Full License 


\section{Abstract}

Background: Current newborn screening (NBS) in China is mainly aimed at detecting biochemical levels of metabolites in blood, which may generate false positive/negative results. Methods: We designed a next-generation sequencing (NGS) panel of 573 genes related to severe diseases and performed NBS for 1173 individuals, who had been screened for tandem mass spectrometry (MS/MS), phenylalanine (Phe), thyroid-stimulating hormone (TSH), 17-a-hydroxyprogesterone (17-OHP) and glucose-6-phosphate dehydrogenase (G6PD) as conducted in traditional biochemical NBS in September 2016. We compared their biochemical results with genetic variants and investigated the carrier frequencies of 77 genes related to disorders of MS/MS in these newborns. Results: Biochemical results showed that four newborns were positive for G6PD enzymatic assay (all males), the other biochemical values including MS/MS, Phe, TSH and 17-OHP were negative. Genetic analysis results showed that all the four newborns with positive G6PD values carried G6PD hemizygous mutations. Besides, NGS results revealed an individual (ID 84123) carrying two common SLC22A5 mutations (c.760C>T/p.R254* and c.1400C>G/p.S467C) in Chinese population which were verified in trans later, was biochemical negative in 2016. MS/MS results in June 2019 showed free carnitine deficiency, which was consistence with genetic analysis results. Carrier frequencies data suggested the top five genes with the highest carrier frequencies in these newborns were PAH (2.04\%), ETFDH (1.24\%), MMACHC (1.15\%), SLC25A13 (0.98\%), and GCDH (0.80\%). Conclusion: Our study provides data that combine biochemical results with genetic variants in 1173 newborns. This is the first study that reveals carrier frequencies of 77 genes causing inherited metabolic diseases (IMDs) in China.

\section{Background}

Newborn screening (NBS) is a successful public health program that prevents morbidity and mortality through early detection and management of specific conditions, it can improve lives of children by allowing timely therapeutic interventions. It is a routine component of neonatal care to screen newborns for inherited metabolic diseases (IMDs) and other common genetic disorders in many countries now. tandem mass spectrometry (MS/MS) is widely used to detect metabolites related to amino acid metabolism, organic acid metabolism and fatty acid oxidation in dried blood spots (DBS). Currently MS/MS is the principal method used in NBS centers to detect biochemical metabolites (1). In China, apart from MS/MS test, traditional biochemical NBS also includes tests for phenylalanine (Phe), thyroidstimulating hormone (TSH), 17-a-hydroxyprogesterone (17-OHP) and glucose-6-phosphate dehydrogenase (G6PD) enzymatic analysis.

The advent of next-generation sequencing (NGS) technologies has enabled the simultaneous and highthroughput detection of genes to identify variants. The rapid development of NGS has reduced the cost of sequencing and genetic analysis to the extent that NGS could be used in NBS. Genome-wide sequencing in the US has been discussed and applied in 159 newborns $(2,3)$, and the results suggested that newborn genomic sequencing can effectively detect the risk and carrier status for a wide range of disorders that are not detectable by current NBS assays (4). Genomic sequencing for newborns can avoid diagnostic 
odyssey for ill newborns, allow optimal early treatment strategies for affected newborns $(5,6)$, and identify carrier status that could help in future reproductive planning. Despite the anticipated benefits, the major challenges hindering its application include data interpretation and appropriate reporting of information. In our previous research, we designed a panel of 77 genes related to over 40 IMDs and demonstrated that genomic DNA extracted from DBS could generate reliable sequencing data, enabling NGS to function as a second-tier diagnostic test for NBS (7).

Considering the benefits and challenges mentioned, we launched a project to screen newborns for genes related to severe diseases. A total of 573 genes related to birth defects were selected based on penetrance, age of onset, and the severity of causal diseases. In total, 1173 newborns that were born in

September 2016 were randomly enrolled in the NBS center of Xinhua Hospital in Shanghai. The newborns had all been screened for MS/MS, Phe, TSH, 17-OHP, and G6PD as in traditional biochemical NBS. The biochemical results and the genetic variants obtained using NGS of the 1173 newborns were compared.

Previous studies showed that the frequency of different Mendelian disorders in China varies with diverse ethnic backgrounds and areas (8). Majority of the newborns recruited in this study were born in Shanghai $(1097,93.52 \%)$, and most of them were of the Han ethnicity, which is the largest ethnic group in China. IMDs, a group of rare diseases with an overall incidence of 1/500 in all ethnic cohorts, are caused by enzyme or transporter abnormalities and alterations in biochemical pathways for metabolism (9). IMDs are genetically heterogeneous disorders and are frequently transmitted in an autosomal recessive inheritance pattern; patients with IMDs may present severe symptoms. In the present study, we investigated the carrier frequencies of IMDs related to amino acid metabolism, organic acid metabolism, and fatty acid oxidation in these random selected newborns.

\section{Methods}

\section{Subjects}

A total of 1173 residual DBS specimens from newborns (600 males and 573 females) at Xinhua Hospital in Shanghai were included in the research. All the 1173 newborns were randomly selected in September 2016 with consecutive number. Among these newborns, 93.52\% (total 1097, 557 males and 540 females) were born in Shanghai and 6.48\% (total 76, 43 males and 33 females) were born in Macao. All the newborns had undergone traditional biochemical NBS, including MS/MS, Phe, TSH, 17-OHP, and G6PD analysis. Written informed consent was obtained from parents/legal guardians. This study reused the NBS samples and was approved by Xinhua Hospital Ethics Committee Affiliated to Shanghai Jiao tong University School of Medicine (XHEC-C-2017-021-2),

\section{Target diseases and genes}

Causative genes for severe diseases including hematological, cardiovascular, rheumatic immunological, gastrointestinal, endocrinological, skeletal, neurological, otolaryngological, muscular, dermatological, 
urinary, respiratory, and IMDs were included. A total of 573 genes related to more than 200 birth defects diseases were selected based on penetrance, age of onset, pathogenicity and mode of inheritance (Additional file 1). Most diseases were inherited recessively, and rare diseases with extremely low incidence rates were excluded.

\section{NGS and data analysis}

Target-specific primers were generated based on the hg19/GRCh37 human reference genome. The primers were designed to have similar length, GC content, and similar amplicon fragment size to maximize the amplification efficiency. The amplicon fragments included the exons and flanking intronic sequences. DNA extracted from a single DBS was generally enough for target capture and library preparation. Amplicons were sequenced on the NovaSeq 6000 instrument (Illumina, San Diego, CA, USA) according to the manufacturer's protocol. Paired-end reads were aligned to the NCBI reference sequence (hg19/GRCh37) using BWA and variant calls were made using GATK. Primary analyses of NGS data included sequence alignment, post-processing, and variant calling.

Variants with allele counts of more than 3 were removed. Allele frequencies lower than 0.005 in ExAC database, gnomAD exome database, and 1000Genomes of East Asian database were further evaluated for pathogenicity according to the ACMG Standards and Guidelines (10). Variants that were selected met either of the following criteria: (1) variants classified as disease-causing mutations in the Human Gene Mutation Database (HGMD); or (2) frameshift, stop-gained, stop-lost, and splice donor/acceptor variants that were supposed to have a high impact on individuals. Traditional biochemical data were compared with the information on genetic variants.

\section{Results}

\section{NGS Assay}

Among the 1173 DBS specimens, genomic DNA extracted from 1127 samples ( 551 females and 576 males) was subjected to quality control and sequencing, while the other 46 DBS failed to meet quality control standards. The average coverage of the target region was $99.97 \%$, and $99.2 \%$ of reads were properly paired and mapped to the reference genome. The mean coverage of sequencing was $305 x$, and $99.4 \%$ of bases were sequenced to at least $20 \times$ coverage.

\section{Traditional biochemical screening compared with genetic variants}

Among these 1173 newborns, there were four newborns (all males, ID:83162, 83841, 83847, 84010) showed low levels of G6PD enzymatic activity (Table 1). MS/MS, Phe and TSH values at birth were negative for all of them. Newborns with G6PD deficiency may suffer from neonatal jaundice and acute 
hemolytic anemia, which is triggered by exogenous primaquine or fava beans (11). G6PD hemizygous variants were detected in all four individuals and the variants were classified as disease-causing mutations according to NGS results. The biochemical values of G6PD were consistent with the information from genetic variants, which indicated the reliable results for G6PD tests.

Besides, there were three newborns with abnormal high levels of 17-OHP at birth and the results turned to be negative when they were called back soon in October 2016 and tested again. This result indicated the false-positives of 17-OHP enzymatic screening.

There were 1173 samples in total subjected to NGS analysis and 1127 samples returned qualified data. Apart from the 4 samples with G6PD hemizygous variants mentioned above, there were 2 individuals with negative biochemical results carrying genetic variants (heterozygous variants for recessively inherited genes were excluded), including one individual with two DUOX2 variants (ID:84066) and one individual with two SLC22A5 variants (ID:84123). In addition, there was one more individual (ID:84193) with two DUOX2 mutations, since integrative genomics viewer showed that the two DUOX2 variants (c.1588A>T/p.K530* and c.1606C>T/p.R536*) were in one allele and were thus not compound heterozygous variants, this case was excluded in Table 1. In April 2019, we contacted the parents of the two children (ID:84066 and 84123) to verify whether the variants were in trans.

We failed to contact the parents of ID 84066. As for ID 84123, SLC22A5 compound heterozygous mutations were verified, including c.760C $>T\left(p . R 254^{\star}\right)$ inherited from his father and c.1400C $>\mathrm{G}(\mathrm{p} . S 467 \mathrm{C})$ inherited from his mother (Fig. 1). The two variants are common mutations in Chinese population. MS/MS was performed for ID 84123 again in May 2019, the results showed free carnitine deficiency, which was consistence with genetic analysis results, indicating false-negative MS/MS result at birth.

\section{Carrier frequencies for IMDs}

The screening population included 1173 newborns with an average age of 3.7 days; $1097(93.52 \%)$ of them were born in Shanghai and the other $76(6.48 \%)$ of them were born in Macao. Among the 1173 DBS specimens, genomic DNA extracted from 1127 (576 males and 551 females) samples were qualified and sequenced. Carrier frequencies of 77 OMIM genes (Additional file 2) related to amino acid metabolism, organic acid metabolism, and fatty acid oxidation were calculated in this study. Variants with allele counts of more than 3 or those with allele frequency higher than 0.005 in ExAC database, gnomAD exome database, and 1000Genomes of East Asian database were excluded. Only variants that were either (1) classified as a disease-causing mutation in HGMD or (2) frameshift, stop-gained, stop-lost, and splice site variants were used to calculate carrier frequencies (Additional file 3). Based on our criteria, 51 genes listed in Table S2 were identified in newborns (Table 2).

\section{Discussion}


The development of NGS revolutionized genetic research and has been proven to be effective for detecting genetic disorders (12). Our previous study has demonstrated that DNA extracted from DBS could be used for NGS; we designed a gene panel incorporating 77 genes related to amino acid metabolism, organic acid metabolism, and fatty acid oxidation, and compared the specificity and sensitivity of the sequencing data (7). This cohort included 1173 DBS, among which 1127 (96.1\%) qualified for NGS. There may be several factors affecting the yield, including the individual differences in white blood cells and DNA degradation rates because the DBS samples had been stored for over a year before being retrieved for NGS. Subjects in our study were born in September 2016, and their NBS samples were continuously numbered from ID 83038 to 84210 .

\section{Comparison of biochemical screening with genetic results}

There were four newborns with low levels of G6PD enzyme and all of them were males that carried hemizygous variants classified as DM in the HGMD database. The biochemical results for G6PD were all consistent with the genetic variants, indicating the high sensitivity and specificity of G6PD. G6PD deficiency is an X-linked, genetic defect due to a mutation in G6PD and is the most common human enzyme defect. The most frequent clinical manifestations of G6PD deficiency are neonatal jaundice and acute hemolytic anemia, which is usually triggered by an exogenous agent. Heterozygous females generally have less severe clinical manifestations than G6PD-deficient males (11). The highest frequencies of G6PD deficiency were detected in tropical Africa, tropical/subtropical Asia, the Mediterranean region, and the Middle East. The global distribution of G6PD deficiency is similar to the epidemic areas of malaria, which provides evidence that G6PD deficiency confers resistance against malaria $(13,14)$. In this study, four male individuals showed low levels of G6PD based on enzymatic assay results, including two males born in Shanghai (ID 83162, 84010) and two males born in Macao (ID $83841,83847)$. In this cohort, 557 males were born in Shanghai, and two of them were identified with G6PD deficiency. While 43 males were born in Macao, which is in the subtropical area of Asia, there were also two males identified with G6PD deficiency. This also indicated the higher prevalence of G6PD deficiency in Macao than in Shanghai.

There were three infants with high levels of 17-OHP at birth, the results turned out to be normal when they were called back soon and tested again in October 2016. The false-positives of 17-OHP enzymatic screening at birth could be time consuming for further reexamination. Thus, a second-tier test may be needed to improve the efficacy of the screening and reduce the number of false-positives.

Apart from the cases mentioned above, there are two children with negative biochemical results carrying DUOX2, and SLC22A5 variants, respectively.

DUOX2 is an autosomal recessive gene related to thyroid hormone synthesis dysfunction (15). There was one case (ID 84066) identified with two DUOX2 variants, c.3391G>T(p.A1131S) and c.2202G>A(p.W734*). Whether the two DUOX2 variants were in the same allele could not be distinguished by integrative genomics viewer, and samples from parents were needed to verify. Our study had an obvious limitation. 
The proband-only sequencing approach makes it hard to distinguish whether the two variants are biallelic or located at one allele of the recessive gene, as we did not have the parents' blood samples, unless the two variants were not far and could be distinguished via integrative genomics viewer.

Homozygous or compound heterozygous mutations in SLC22A5 can cause systemic primary carnitine deficiency (SPCD) and lead to impaired fatty acid oxidation in muscles (16). There was one case (ID 84123) with SLC22A5 compound heterozygous variants. His CO (free carnitine) value was $11.6 \mathrm{umol} / \mathrm{L}$ (reference: 10-60umol/L) at birth in September 2016. When he was called back in April 2019 due to $S L C 22 A 5$ mutations, the $C 0$ value was $4.3 \mathrm{umol} / \mathrm{L}$, which was lower than normal range and could be classified into SPCD. SPCD encompasses a broad clinical spectrum including metabolic decompensation in infancy; childhood myopathy involving heart and skeletal muscle; pregnancy-related decreased stamina or exacerbation of cardiac arrhythmia; fatigability in adulthood or absence of symptoms (17). Genomic screening can avoid diagnostic odyssey and allow optimal early treatment strategies for affected newborns. The child is near 3 years old now, he has not shown symptoms yet. It is important for this child to have physical examination regularly and possible treatment to maintain plasma carnitine and prevent primary manifestations.

\section{Carrier frequencies}

Our team focused on IMDs, and we investigated the carrier frequencies of 77 genes related to disorders of MS/MS including amino acid metabolism, organic acid metabolism and fatty acid oxidation, which are listed in Table S2. A total of 51 genes were identified in 1127 subjects, and the other 26 genes with LP/P variants were not detected according to our criteria. The top five genes with the highest carrier frequencies in these newborns were PAH (2.04\%), ETFDH (1.24\%), MMACHC (1.15\%), SLC25A13 (0.98\%), and $G C D H(0.80 \%)$. This is the first study that reveals the carrier frequencies of this many genes in China.

In 2018, a pilot study of expanded carrier screening in China investigated 11 recessive diseases, including $P A H$ deficiency, and revealed that the carrier frequency for $P A H$ in all ethnicities in China was $3.59 \%$. The results varied among different ethnicities and the carrier frequency for $P A H$ in the Han ethnicity was $3.30 \%$ (8), which was higher than what was calculated in our study $(2.04 \%)$. The subjects in our study were mainly born in Shanghai and the population was mostly of the Han ethnicity. One of the reasons for the varying carrier frequencies obtained in the two studies may be the different criteria employed for determining variants.

Another study in 2016 performed a molecular approach for NBS for four genetic diseases in Guizhou Province of south China, including beta-thalassemia, G6PD deficiency, PKU, and non-syndromic hearing loss. They included 515 newborns and selected 10 common mutations of $P A H$ in the Chinese population, and the carrier frequency for PAH they calculated in their study was $0.78 \%(18)$. The frequency they calculated was much lower than that in our study (2.04\%). Our data indicated 23 newborns carrying variants with mutations in 18 different positions, and all 18 variants were classified as disease-causing 
mutations. This comparison also suggests that $P A H$ is better screened as whole exons instead of as variants based on mutations in several positions.

\section{Conclusions}

Our study provide data that combine biochemical results with genetic variants of newborns, which could be helpful for the evaluation of other expanded NBS strategies. We identified an individual (ID 84123)carrying two common SLC22A5 mutations (c.760C>T/p.R254* and c.1400C>G/p.S467C) in Chinese population which were verified in trans later, was biochemical negative in 2016. MS/MS results in June 2019 showed free carnitine deficiency, which was consistence with genetic analysis results. This is also the first study that reveals carrier frequencies of 77 genes causing IMDs related to amino acid metabolism, organic acid metabolism, and fatty acid oxidation in China.

\section{Abbreviations}

NGS: next-generation sequencing; NBS: newborn screening; MS/MS: tandem mass spectrometry; Phe: phenylalanine, TSH: thyroid-stimulating hormone; 17-OHP: 17-a-hydroxyprogesterone; G6PD: glucose-6phosphate dehydrogenase; IMDs: inherited metabolic diseases; DBS: dried blood spots; SPCD: systemic primary carnitine deficiency.

\section{Declarations}

\section{Ethics approval and consent to participate}

This study reused the NBS samples and was approved by Xinhua Hospital Ethics Committee Affiliated to Shanghai Jiao tong University School of Medicine (XHEC-C-2017-021-2). Written informed consent was obtained from parents/legal guardians.

\section{Consent for publication}

Not applicable

\section{Availability of data and materials}

The data generated or analyzed during this study are included in this article and its supplementary files.

\section{Competing interests}

The authors declare that they have no conflict ofinterest. 


\section{Funding}

This work was supported by the National Key R\&D Program of China (2018YFC1002204 to YGY); the National Natural Science Foundation of China (81670812 and 81873671 to YGY); the Jiaotong University Cross Biomedical Engineering (YG2017MS72 to YGY); the Shanghai Municipal Commission of Health and Family Planning (201740192 to YGY and 20184Y0073 to XML); the Shanghai Shen Kang Hospital Development Center new frontier technology joint project (SHDC12017109 to YGY); the Shanghai Science and Technology Commission (19140904500 to YGY). The funders had no role in the study design, data collection, data analysis, interpretation of results, and writing of the manuscript.

\section{Author contributions}

$X G$ and $Y Y$ conceived and designed the study. FX, JG and JY provided biochemical results of all the DBS samples. LL and ZL performed the NGS experiments. YS interpreted the NGS data. XL analyzed the sequencing data and drafted the manuscript. All authors reviewed and approved the final manuscript.

\section{Acknowledgments}

We thank the families and clinicians for their participation in this project.

\section{Authors' Information}

${ }^{1}$ Department of Pediatric Endocrinology/Genetics, Xinhua Hospital, Shanghai Jiao Tong University School of Medicine, Shanghai, China, 200092.

${ }^{2}$ Shanghai Institute for Pediatric Research, Shanghai, China, 200092.

${ }^{3}$ Nanjing Novogene Bio Technology Co., Ltd. Nanjing, China, 210000

\section{References}

1.Ombrone D, Giocaliere E, Forni G, Malvagia S, la Marca G. Expanded newborn screening by mass spectrometry: New tests, future perspectives. Mass Spectrom Rev. 2016;35(1):71-84.

2.Holm IA, Agrawal PB, Ceyhan-Birsoy O, Christensen KD, Fayer S, Frankel LA, et al. The BabySeq project: implementing genomic sequencing in newborns. BMC pediatrics. 2018;18(1):225.

3.Berg JS, Agrawal PB, Bailey DB, Jr., Beggs AH, Brenner SE, Brower AM, et al. Newborn Sequencing in Genomic Medicine and Public Health. Pediatrics. 2017;139(2). 
4.Ceyhan-Birsoy O, Murry JB, Machini K, Lebo MS, Yu TW, Fayer S, et al. Interpretation of Genomic Sequencing Results in Healthy and III Newborns: Results from the BabySeq Project. Am J Hum Genet. 2019;104(1):76-93.

5.Meng L, Pammi M, Saronwala A, Magoulas P, Ghazi AR, Vetrini F, et al. Use of Exome Sequencing for Infants in Intensive Care Units: Ascertainment of Severe Single-Gene Disorders and Effect on Medical Management. JAMA Pediatr. 2017;171(12):e173438.

6.Petrikin JE, Cakici JA, Clark MM, Willig LK, Sweeney NM, Farrow EG, et al. The NSIGHT1-randomized controlled trial: rapid whole-genome sequencing for accelerated etiologic diagnosis in critically ill infants. NPJ Genom Med. 2018;3:6.

7.Luo X, Wang R, Fan Y, Gu X, Yu Y. Next-generation sequencing as a second-tier diagnostic test for newborn screening. Journal of pediatric endocrinology \&amp; metabolism: JPEM. 2018;31(8):927-31.

8.Zhao S, Xiang J, Fan C, Asan, Shang X, Zhang X, et al. Pilot study of expanded carrier screening for 11 recessive diseases in China: results from 10,476 ethnically diverse couples. Eur J Hum Genet. $2019 ; 27(2): 254-62$.

9.Feuchtbaum L, Carter J, Dowray S, Currier RJ, Lorey F. Birth prevalence of disorders detectable through newborn screening by race/ethnicity. Genet Med. 2012;14(11):937-45.

10.Richards S, Aziz N, Bale S, Bick D, Das S, Gastier-Foster J, et al. Standards and guidelines for the interpretation of sequence variants: a joint consensus recommendation of the American College of Medical Genetics and Genomics and the Association for Molecular Pathology. Genet Med. $2015 ; 17(5): 405-24$.

11.Cappellini MD, Fiorelli G. Glucose-6-phosphate dehydrogenase deficiency. Lancet. 2008;371(9606):64-74.

12.Boycott KM, Vanstone MR, Bulman DE, MacKenzie AE. Rare-disease genetics in the era of nextgeneration sequencing: discovery to translation. Nat Rev Genet. 2013;14(10):681-91.

13.Ruwende C, Hill A. Glucose-6-phosphate dehydrogenase deficiency and malaria. J Mol Med. 1998;76(8):581-8.

14.Kotepui M, Uthaisar K, PhunPhuech B, Phiwklam N. Prevalence and hematological indicators of G6PD deficiency in malaria-infected patients. Infect Dis Poverty. 2016;5:36.

15.Srichomkwun P, Takamatsu J, Nickerson DA, Bamshad MJ, Chong JX, Refetoff S. DUOX2 Gene Mutation Manifesting as Resistance to Thyrotropin Phenotype. Thyroid. 2017;27(1):129-31.

16.Wang Y, Taroni F, Garavaglia B, Longo N. Functional analysis of mutations in the OCTN2 transporter causing primary carnitine deficiency: lack of genotype-phenotype correlation. Hum Mutat. 
2000;16(5):401-7.

17.El-Hattab AW. Systemic Primary Carnitine Deficiency. In: Adam MP, Ardinger HH, Pagon RA, Wallace SE, Bean LJH, Stephens K, et al., editors. GeneReviews((R)). Seattle (WA): University of Washington, Seattle niversity of Washington, Seattle. GeneReviews is a registered trademark of the University of Washington, Seattle. All rights reserved.; 1993.

18.Huang S, Xu Y, Liu X, Zhou M, Wu X, Jia Y. Molecular newborn screening of four genetic diseases in Guizhou Province of South China. Gene. 2016;591(1):119-22.

\section{Tables}

Table 1 Biochemical and genetic results of the newborns

\begin{tabular}{|c|c|c|c|c|c|c|c|c|}
\hline \multirow[t]{2}{*}{ ID } & \multirow[t]{2}{*}{ Sex } & \multicolumn{5}{|c|}{ Biochemical results" } & \multirow[t]{2}{*}{ Genetic Variants* } & \multirow[t]{2}{*}{ Zygosity } \\
\hline & & MS & Phe & $\overline{\mathrm{TSH}}$ & $\begin{array}{l}17- \\
\text { OHP }\end{array}$ & G6PD & & \\
\hline 83162 & $\bar{M}$ & $\mathrm{~N}$ & $\mathrm{~N}$ & $\mathrm{~N}$ & $\mathrm{~N}$ & $\mathbf{P}$ & G6PD c.1114C>T(p.L372F) & Hemizygous \\
\hline 83841 & M & $\mathrm{N}$ & $\mathrm{N}$ & $\mathrm{N}$ & $\mathrm{N}$ & $\mathbf{P}$ & G6PD c.1478G>A(p.R493H) & Hemizygous \\
\hline 83847 & M & $\mathrm{N}$ & $\mathrm{N}$ & $\mathrm{N}$ & $\mathrm{N}$ & $\mathbf{P}$ & G6PD c.1478G>A(p.R493H) & Hemizygous \\
\hline 84010 & M & $\mathrm{N}$ & $\mathrm{N}$ & $\mathrm{N}$ & $\mathrm{N}$ & $\mathbf{P}$ & G6PD c.496C>T(p.R166C) & Hemizygous \\
\hline $84066^{+}$ & $\mathrm{F}$ & $\mathrm{N}$ & $\mathrm{N}$ & $\mathrm{N}$ & $\mathrm{N}$ & $\mathrm{N}$ & $\begin{array}{l}D U O X 2 \text { c. } 3391 \mathrm{G}>\mathrm{T}(\mathrm{p} . \mathrm{A} 1131 \mathrm{~S}), \\
\text { c.2202G }>\mathrm{A}\left(\mathrm{p} . \mathrm{W} 734^{*}\right)\end{array}$ & unknown \\
\hline $84123^{++}$ & M & $\mathbf{P}$ & $\mathrm{N}$ & $\mathrm{N}$ & $\mathrm{N}$ & $\mathrm{N}$ & $\begin{array}{l}S L C 22 A 5 \text { c. } 760 \mathrm{C}>\mathrm{T}\left(\mathrm{p} . \mathrm{R} 254^{*}\right), \\
\text { c. } 1400 \mathrm{C}>\mathrm{G}(\mathrm{p} . \mathrm{S} 467 \mathrm{C})\end{array}$ & $\begin{array}{l}\text { Compound } \\
\text { heterozygous }\end{array}$ \\
\hline
\end{tabular}

Abbreviations: $\mathrm{MS}=\mathrm{MS} / \mathrm{MS}, \mathrm{M}=$ Male, $\mathrm{F}=$ Female, $\mathrm{N}=$ Negative, $\mathrm{P}=$ Positive,

Transcripts: $G 6 P D$ (NM_000402.4), DUOX2(NM_014080.4), SLC22A5(NM_003060.3)

\#There were three more newborns with abnormal levels of 17-OHP at birth which are not listed in the table because the 17-OHP results turned to be negative when they were called back soon and tested again.

*There was one more newborn (ID 84193) with two DUOX2 variants, integrative genomics viewer showed that the two variants were in one allele and were thus excluded in the table.

+Parents of this individual could not be contacted.

${ }^{++}$The MS/MS test for ID 84123 at birth was negative, but the plasma free carnitine value turned to be positive when he was called back in April 2019 to confirm the data.

Table 2 Carrier frequencies of 77 genes causing IMDs in the cohort of 1127 newborns 


\begin{tabular}{|c|c|c|c|c|}
\hline Disease & Gene & $\mathrm{N}$ & $\%$ & $1 \mathrm{in}_{-}$ \\
\hline \multirow[t]{3}{*}{ Hyperphenylalaninemia } & $P A H$ & 23 & 2.04 & 49 \\
\hline & PTS & 6 & 0.53 & 188 \\
\hline & GCH1 & 2 & 0.18 & 564 \\
\hline Sepiapterin reductase deficiency & $S P R$ & 1 & 0.09 & 1127 \\
\hline Maple syrup urine disease & $B C K D H B$ & 2 & 0.18 & 564 \\
\hline Carbamoyl phosphate synthetase I deficiency & CPS1 & 4 & 0.35 & 282 \\
\hline Ornithine transcarbamylase deficiency & OTC & 1 & 0.09 & 1127 \\
\hline \multirow[t]{2}{*}{ Citrullinemia } & $A S S 1$ & 4 & 0.35 & 282 \\
\hline & $S L C 25 A 13$ & 11 & 0.98 & 102 \\
\hline Argininosuccinic aciduria & $A S L$ & 2 & 0.18 & 564 \\
\hline Argininemia & $A R G 1$ & 5 & 0.44 & 225 \\
\hline Hyperornithinemia-hyperammonemia-homocitrullinuria syndrome & $S L C 25 A 15$ & 1 & 0.09 & 1127 \\
\hline Ornithine aminotransferase deficiency & $O A T$ & 1 & 0.09 & 1127 \\
\hline \multirow{2}{*}{ Nonketotic hyperglycinemia } & $G L D C$ & 3 & 0.27 & 376 \\
\hline & $A M T$ & 3 & 0.27 & 376 \\
\hline Homocystinuria & $C B S$ & 3 & 0.27 & 376 \\
\hline Methylenetetrahydrofolate reductase deficiency & MTHFR & 3 & 0.27 & 376 \\
\hline Methionine synthase deficiency & $M T R$ & 1 & 0.09 & 1127 \\
\hline Methionine synthase reductase deficiency & $M T R R$ & 1 & 0.09 & 1127 \\
\hline Hypermethioninemia & MAT1A & 2 & 0.18 & 564 \\
\hline$S$-adenosylhomocysteine hydrolase deficiency & $A H C Y$ & 2 & 0.18 & 564 \\
\hline \multirow{4}{*}{ Methylmalonic aciduria } & $M U T$ & 8 & 0.71 & 141 \\
\hline & $M M A A$ & 2 & 0.18 & 564 \\
\hline & $M M A B$ & 1 & 0.09 & 1127 \\
\hline & $M M A C H C$ & 13 & 1.15 & 87 \\
\hline \multirow[t]{2}{*}{ Propionic acidemia } & $P C C A$ & 2 & 0.18 & 564 \\
\hline & $P C C B$ & 1 & 0.09 & 1127 \\
\hline Isovaleric acidemia & IVD & 1 & 0.09 & 1127 \\
\hline \multirow[t]{2}{*}{ Glutaric aciduria } & $G C D H$ & 9 & 0.80 & 125 \\
\hline & $S U G C T$ & 2 & 0.18 & 564 \\
\hline \multirow[t]{2}{*}{ 3-Methylcrotonyl-CoA carboxylase deficiency } & $M C C C 1$ & 2 & 0.18 & 564 \\
\hline & MCCC2 & 3 & 0.27 & 376 \\
\hline Holocarboxylase synthetase deficiency & $H L C S$ & 3 & 0.27 & 376 \\
\hline Biotinidase deficiency & $B T D$ & 8 & 0.71 & 141 \\
\hline HMG-CoA lyase deficiency & $H M G C L$ & 2 & 0.18 & 564 \\
\hline Alpha-methylacetoacetic aciduria & $A C A T 1$ & 1 & 0.09 & 1127 \\
\hline Malonyl-CoA decarboxylase deficiency & $M L Y C D$ & 1 & 0.09 & 1127 \\
\hline 2-Methylbutyrylglycinuria & $A C A D S B$ & 4 & 0.35 & 282 \\
\hline Isobutyryl-CoA dehydrogenase deficiency & $A C A D 8$ & 6 & 0.53 & 188 \\
\hline Succinic semialdehyde dehydrogenase deficiency & $A L D H 5 A 1$ & 4 & 0.35 & 282 \\
\hline Mevalonic aciduria & $M V K$ & 2 & 0.18 & 564 \\
\hline HMG-CoA synthase-2 deficiency & $H M G C S 2$ & 2 & 0.18 & 564 \\
\hline Primary carnitine deficiency & $S L C 22 A 5$ & 8 & 0.71 & 141 \\
\hline \multirow[t]{2}{*}{ Carnitine palmitoyltransferase deficiency } & CPT1A & 1 & 0.09 & 1127 \\
\hline & CPT2 & 2 & 0.18 & 564 \\
\hline
\end{tabular}


Short-chain acyl-CoA dehydrogenase deficiency Medium-chain acyl-CoA dehydrogenase deficiency

Very long-chain acyl-CoA dehydrogenase deficiency

3-Hydroxyacyl-CoA dehydrogenase deficiency

Trifunctional protein deficiency

Other acyl-CoA dehydrogenase deficiency diseases
$\begin{array}{lll}A C A D S & 7 & 0.62\end{array}$

$\begin{array}{llll}A C A D M & 7 & 0.62 & 161\end{array}$

The other 26 genes were not detected in the cohorts: $Q D P R, P C B D 1, B C K D H A, D B T, D L D, F A H, T A T, H P D$, GCSH, HGD, MMADHC, MCEE, AUH, TAZ, OPA3, DNAJC19, ASPA, ETHE1, L2HGDH, D2HGDH, IDH2, SLC25A20, ETFA, ETFB, DECR1, HADHA.

\section{Figures}

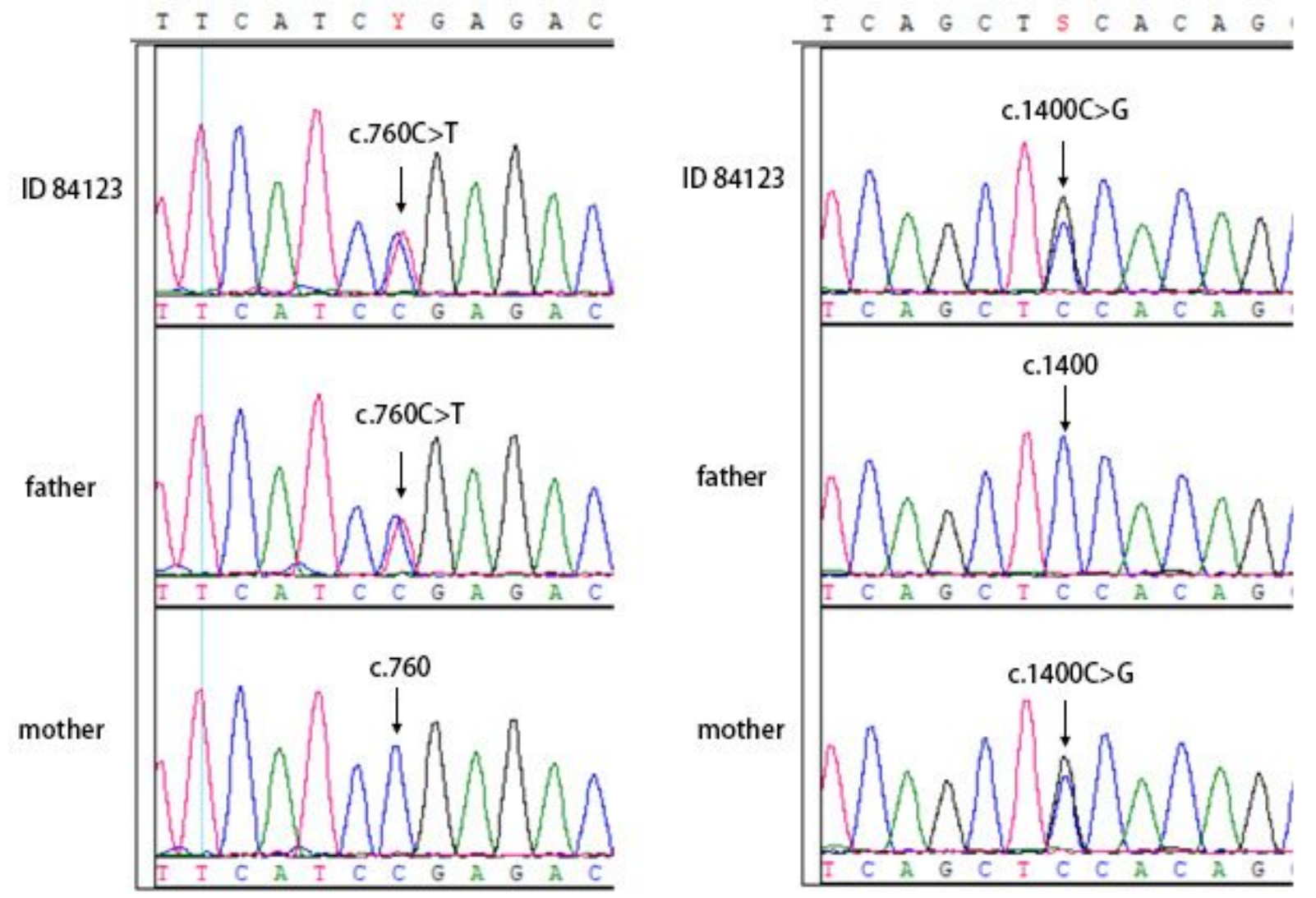

\section{Figure 1}

Sequencing analysis of the SLC22A5 gene mutations. The arrows show the site of the c.760 and c.1400 of the family. 


\section{Supplementary Files}

This is a list of supplementary files associated with this preprint. Click to download.

- supplement1.xlsx

- supplement2.docx

- supplement3.xIsx 